\title{
Persuasive e-commerce website design assistant tool
}

\author{
Idyawati H., E. A. Seman \& Y. Yusak \\ University Malaysia Sabah, Malaysia
}

\begin{abstract}
e-Commerce websites that are not structured or planned can convey the wrong message to users at best and at worse are the cause of fatigue and stress. A poorly-constructed interface will repel users, lose sales, do damage to a company's brand, and simply become an irrelevance to the business. An excess of visual, text and/or audio information burdens the user, who in turn will discard the underlying messages that the site is attempting to convey. The success of a well designed e-commerce websites can be measured through the experience of a user, and the information the user imparts from the event. The aim, therefore, of any multimedia designer especially a persuasive e-commerce website designer is to understand the needs of any potential user to effectively convey the central themes of the design and promote comprehension of the services/products/information on display.
\end{abstract}

Keywords: paradigm shift, cognitive model, human information processing, comprehension, create creativity, psychological knowledge, multi-sensory interaction.

\section{Introduction}

This paper is intended to create a tool, which is called the Multimedia Design Assistant Tool (MDAT) which consists of Cognitive Advisor Tool (CAT) to shift designer's paradigm in promoting a persuasive e-commerce website. The tool reflects a designer's perception of how to utilize multimedia and hypermedia in such a way that they are optimising the aesthetic characteristics of a particular media. The tool itself will be created in a user-centred and interactive manner to ensure that designers are actively engaged in the environment rather than passively receiving and storing knowledge from teachers and textbooks. 
The growing awareness in practical necessities in serving users with slow and fast network connections, small and large displays, and fast and slow computers leads to shifting the paradigm of designers towards improving e-commerce and information systems. Apparently, the industrial designer has one central interest to consider when he or she is developing an e-commerce website: the interest of the client or a company [9].

The need and motivation for human factors to support designers' creativity is often pointed out, and Ben Shneiderman [7] argues that: The enormous interest in Human Factors of interactive arises from the complementary recognition of how poorly designed systems are and how genuinely developers desire to create elegant systems that serve the users effectively. Human factors competence in product design consists of information and knowledge about the user and the methods and techniques to bring this knowledge into the design process.

One aspect of design is still overlooked by the industrial designers; while operative usability is rightly considered to be an essential part of design, the aesthetic appeal of any application is an equally important consideration for users in judging if they firstly want to use the application and secondly in deciding how much time they will spend on the aforementioned application.

Taking into consideration the various disciplines required for multimedia design, the ideal application should balance aesthetics with usability if the programmer is to achieve the aim of creating a satisfying user experience. Ideally, the basics of design theory and research can be studied by programmers who are looking to create more intuitive e-commerce website, particularly from the design view of the user interface.

However, in practice the time limitations imposed on designers leads researchers such as Sutcliffe [8] to assert that if the knowledge of the workings of the user cognitive model regarding multimedia information processing cannot keep pace with the requirements of the design community, then an alternative is to deliver usability via examples of good practice and/or reusable artefacts. He illustrates 'claims' as psychologically motivated design rationales that express the upsides and downsides of a design as a usability issue. These 'claims' therefore encourage designers to consider a trade-off in respects to the various multimedia design methods rather than accepting a single guideline or principle.

An understanding of the multimedia design process - from a cognitive perspective - and awareness of the gaps that exist in the experience of the design industry will allow the researcher to understand how to best assist the designer and his/her psychological knowledge in relation to design. The purpose of this is to aid the designer in effectively investing the time and resources into good practices of design.

\section{Problem recognition}

The background of the problem is when the designer has to select which type of media to be used to create an effective attention directing persuasive e-commerce website. The layout of the media must be taken into account. For example, one text and two or more animations on a screen translates to one paragraph of text 
with two or more animations displayed concurrently. The media types are perceived via a variety of senses, but memory plays a crucial role in interpreting these media. This task involves many subtasks.

Cognitive psychology describes multimedia in terms of human information processing as shown in Figure 1. In the structural perspective, input is coded and stored in the short or long-term memory. Input is gained by the 'human processor' through the sensory organs - in this case, the eye. A visual sensory register, the retina, then converts this input from an electromagnetic spectrum to an electrical signal, or 'pattern'.

Pattern recognition in the brain then checks for matches between the input patterns and patterns held in the long-term memory. This process is dependent on attention and motivation. It is this factor which leads cognitive psychologists to suggest control processes which influence 'memorisation'. These include motivation, retrieval and, most importantly, memory.



Figure 1: Visual inputs for model of human information processing.

In this model a pattern may be committed to long-term memory after rehearsal in the short-term memory. Patterns will be committed to memory most effectively if the task at hand holds the attention. In figure 1, the input pattern is identified as text and image. The user's vision moves around the image and try to interpret the objects. If the image is still less predictable, then, the user's viewing will attend to text component, although no guarantee can be given that both components are related to each other. However, the attention-directing design effects can increase the probability that the user will attend to the image component first.

Paying attention to input, repetition or rehearsal of input and grouping of input into coherent and easily remembered categories may all contribute to 
improve recall [4]. Animation and video tends to dominate attention over static text and image. In multimedia, memorisation fails because an access path either decays through lack of use or was poorly constructed in the first place. This happens when two or more dynamic media are played concurrently.

The combination of media is determined by how it is displayed in adjacent windows. According to Multimedia Design Guidelines from ISO 14915, Part 3, two or more media are considered combined if their presentation is concurrent or contiguous when the media are explicitly grouped in a display. To select the media type, consisting of text, image, audio and animation/video, a designer must know the cognitive psychology for human information processing involved for each media type.

The cognitive models are useful as the models explain how perception and comprehension are related. Then, after acquiring such knowledge from the Multimedia Design Assistant Tool (MDAT), the designer will be able to make a decision as to which type of attention directing effect should be taken into consideration for delivering the important message he/she wants to convey.

\section{Use case diagram analysis and design}

The main actor for the MDAT is a website designer: They search for the advisor; select media type and get guidelines according to media types. In addition, they may choose an interactive example and get interactive guidelines from the advisor.

The objective of the use case model is to document what functions the system should offer to the user. The purpose is to construct the developer's view of what the users want, to provide a starting point for discovering the object classes and for discovering the operations for each class. The focus of the use cases is on the usage of the system, rather than on the users themselves. The use cases provide an outside view of the system and the services and behaviours and responses the system must provide.

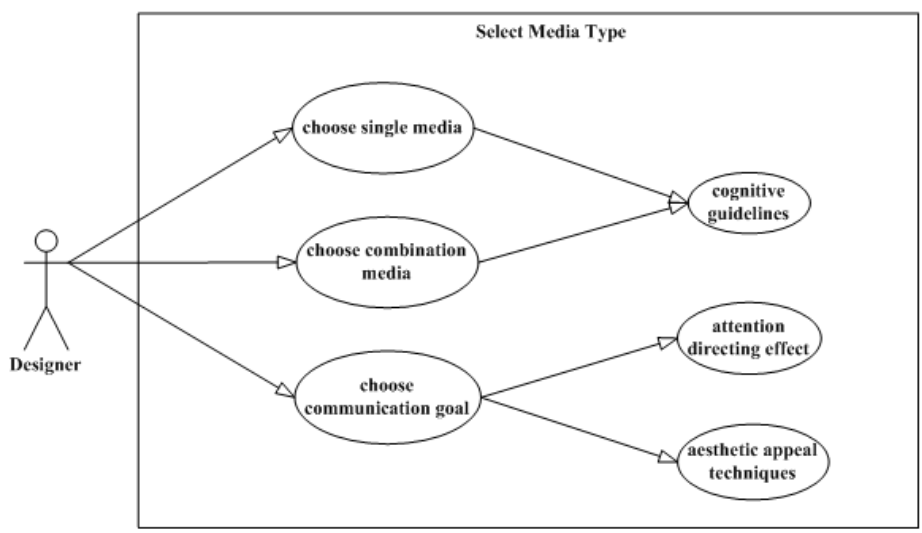

Figure 2: Designer's media selection task. 
Figure 2 shows the use case for the designer. The use case for MDAT is divided into two categories, which are cognitive advisor and attention advisor. Below is the use case of the designer getting the cognitive guidelines from the MDAT. The tasks that designers are able to select include that of either a single media type or a combination of media to get cognitive guidelines. They could also select the media type after choosing types of communication goals for the attention directing guidelines.

Table 1: $\quad$ Use case description and functionality.

\begin{tabular}{|c|c|}
\hline Use Case & Select Advisor \\
\hline Actor & Designer \\
\hline Description & $\begin{array}{l}\text { This use case begins when a user requests to use the tool. } \\
\text { The user will choose to use the cognitive advisor or } \\
\text { attention advisor tool. The MDAT will provide a } \\
\text { standardized interface for the user to choose level of skills } \\
\text { by checking the "Learning Mode" at the main menu. }\end{array}$ \\
\hline $\begin{array}{l}\text { Remarks and } \\
\text { constraints }\end{array}$ & $\begin{array}{l}\text { If the user does not check on the "Learning Mode", the } \\
\text { tool will generate the normal window that does not have } \\
\text { additional information and step-by-step instructions on } \\
\text { how to use the tool. }\end{array}$ \\
\hline Use Case & Cognitive Advisor \\
\hline Actor & Designer \\
\hline Description & $\begin{array}{l}\text { This use case begins when a user selects the cognitive } \\
\text { advisor tool from the main menu. The tool allows the user } \\
\text { to enter media type by selecting from a list of single media } \\
\text { type or list of combination media from combo box } \\
\text { available. The tool will activate the model of human } \\
\text { information processing component and guidelines } \\
\text { accordingly. }\end{array}$ \\
\hline $\begin{array}{l}\text { Remarks and } \\
\text { constraints }\end{array}$ & $\begin{array}{l}\text { If the user selects combination media type, the single } \\
\text { media type will be disabled from any event. There will be } \\
\text { no event occurrence if the user clicks on the single media } \\
\text { type after selecting the combination media type. The } \\
\text { "Reset" button can be chosen to return to the default value } \\
\text { of the tool. }\end{array}$ \\
\hline Use Case & Attention Advisor \\
\hline Actors & Designer \\
\hline Description & $\begin{array}{l}\text { This use case begins when a user selects the attention } \\
\text { advisor tool from the main menu of the tool. The user will } \\
\text { need to choose communication goals of the application to } \\
\text { get guidelines for each media type. When a user selects } \\
\text { one of the option buttons, the tool will activate media type } \\
\text { for guidelines. }\end{array}$ \\
\hline
\end{tabular}


Table 1: Continued.

\begin{tabular}{|c|c|}
\hline $\begin{array}{l}\text { Remarks and } \\
\text { constraints }\end{array}$ & $\begin{array}{l}\text { The user can choose to get an interactive guideline and } \\
\text { example from the "Interactive Guidelines" command } \\
\text { button whenever he/she wishes to do so. }\end{array}$ \\
\hline Use Case & Select Media Type \\
\hline Actors & Designer \\
\hline Description & $\begin{array}{l}\text { This use case begins when a user wishes to get guidelines } \\
\text { on the cognitive advisor tool. The media type consists of: } \\
\text { - Text } \\
\text { - Image } \\
\text { - Audio } \\
\text { - Animation/Video } \\
\text { The user enters the media type and the cognitive advisor } \\
\text { tool will activate components on the Model of Human } \\
\text { Information Processing and show guidelines in a } \\
\text { descriptive manner. }\end{array}$ \\
\hline $\begin{array}{l}\text { Remarks and } \\
\text { constraints }\end{array}$ & $\begin{array}{l}\text { The user cannot select single media type if he/she has } \\
\text { already selected combination media type for cognitive } \\
\text { advisor tool. The "Reset" button helps the user to reset the } \\
\text { current event of the tool to default values. The Attention } \\
\text { advisor tool provides two communication goals for the } \\
\text { user to select in order to activate the media type button. }\end{array}$ \\
\hline Use Case & View Guidelines \\
\hline Actors & Designer \\
\hline Description & $\begin{array}{l}\text { This use case begins when a user selects a media type from } \\
\text { both advisors. The guidelines are described in text media. } \\
\text { However, there is an interactive presentation of guidelines } \\
\text { available on the attention advisor tool. }\end{array}$ \\
\hline $\begin{array}{l}\text { Remarks and } \\
\text { constraints }\end{array}$ & $\begin{array}{l}\text { The guidelines are filtered by media type selected by the } \\
\text { user. }\end{array}$ \\
\hline
\end{tabular}

\section{Conceptual design}

The use case begins when the designer requests for the advisor tools for the MDAT. There are two types of advisors available: Cognitive Advisor Tool and Attention Advisor Tool. Both tools will be displayed in a different screen layout, consisting of different designs and scenarios of usage. If the user selects the Cognitive Advisor Tool, the next screen will display the media types, their combinations, and components of human information processing involves for the media type selected. Each media will result in different components for the model of human information processing and guidelines. A highlighting 
technique should be used to present which component of Model of Information Processing (MHIP) is involved for the media type selected by the designer.

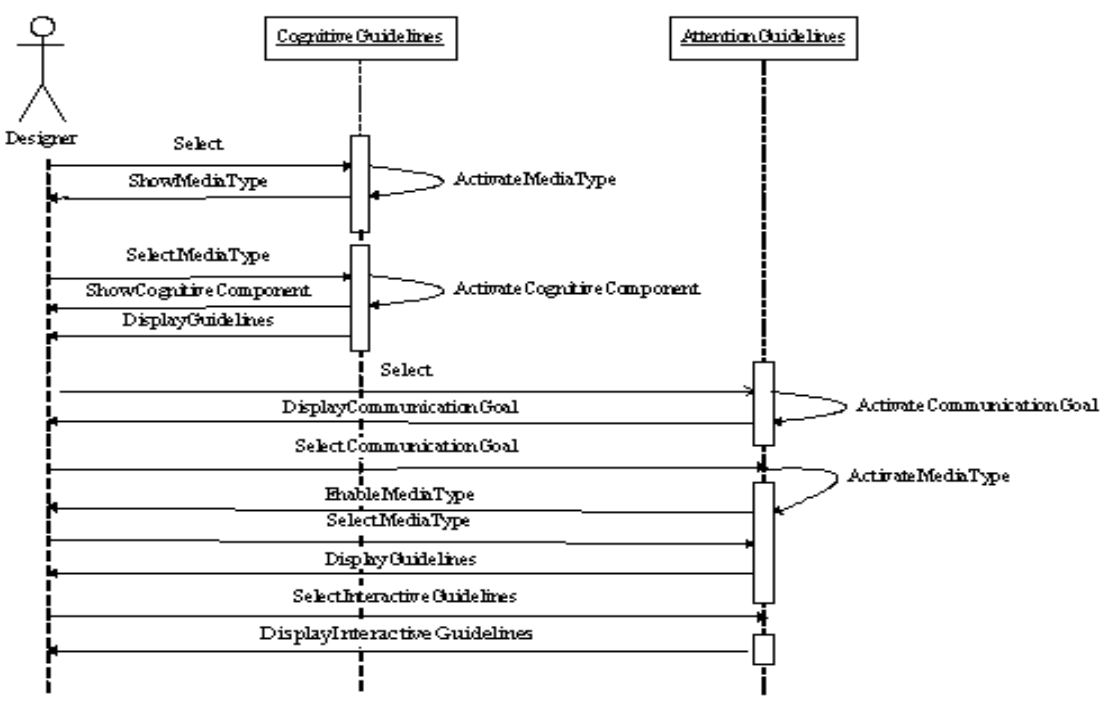

Figure 3: Use case interaction diagram for MDAT.

If the user selects the Attention Advisor Tool, the next task will be selecting the communication goal for the application. In this tool, there are only two communication goals available because the focus of the MDAT is to create an aesthetic appealing and attention attracting application: Attention Directing Effect and Aesthetic Appeal Guidelines to persuade users to browse thru the e-commerce website. Each media type will be activated according to communication goal.

The guidelines will be displayed on the provided layout. The interactive guidelines will present guidelines and example in an interactive manner. This will only be available by clicking on to a command button "Interactive Guidelines" on the screen. The scenario steps will be placed on the sequence diagram as a first draft preparation for the design. The sequence diagram is chosen because it identifies the operations that respond to each step involved in the MDAT. These sequences are collected during the contextual design to reflect the designer's work sequence. Many messages will be sent to and from the designers as usage of the MDAT progresses.

To get the cognitive guidelines, the designer needs to choose a particular media type that will be used in the design. Then, the cognitive advisor tool will display the information-processing model that will provide guidelines associated with the media. The attention-directing advisor consists of attention directing techniques and aesthetic appealing guidelines. These guidelines will be displayed after the designer chooses the communication goals from the attention advisor 
tool. The guidelines will be filtered by the media type selected by the designer. For example, text will have few attentions directing techniques on text fonts such as bold, underline, flash and revealing captions. Each attention directing technique will be shown according to the media selected. The following figure shows the sequence diagram of the MDAT.

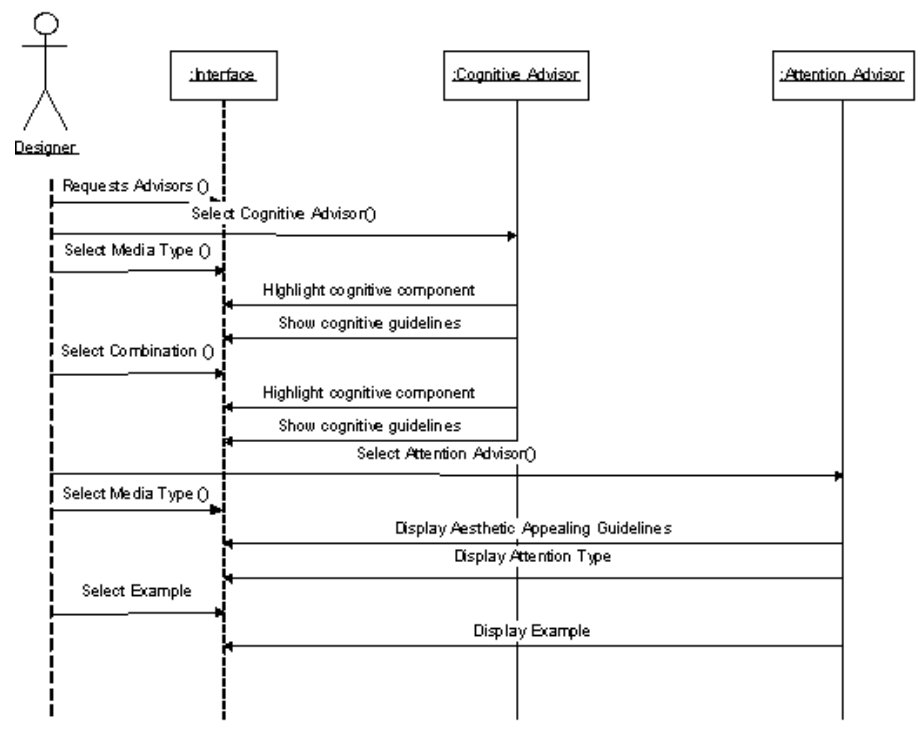

Figure 4: Sequence diagram with operation of MDAT.

The first screen shot of the MDAT consists of media type object and the display of MHIP. The designer can select the media type for his/her application from the media type drop down list box and select the media type.

There are four types of media available: Text, Image, Audio and Animation/Video. For each selection, the MHIP will display the category of input modality and information processing necessary for the media. For example: selecting 'Text' will highlight the eye since text is a perceptual data, which is seen through vision. The cognitive processor and working memory object will be highlighted as these models are involved in processing information that is retrieved by human vision.

The tool will show which component of the MHIP is involved for the media type selection. As can be seen from the main interface of the Cognitive Advisor tool, the component consists of:
1) Audio/Visual Short Term Memory
2) Cognitive Processor,
3) Attention Schedule,
4) Working Memory and
5) Long Term Memory
6) Bottleneck 
Single media type like text, image and animation/video are perceived via eyes and the information will be passed into the visual short term memory. In this case, the MHIP will activate the component involves which is visual short term memory and display advice in accordance to cognitive guidelines.

However, combining media can also help accommodate user preferences for information in a particular format. Therefore, the cognitive advisor will provide guidelines for media combination that map the human mental activity to appropriate media types for effective delivery. Failure to choose the right combination media will result bottleneck for each information-processing component.

For the Cognitive Advisor tool, the bottleneck guidelines will be highlighted if the combination of media is not recommended. To complete the model, the cognitive guidelines will be displayed at the bottom of the model. Each component can be clicked to get further information and explanation.

If the user selects single media from the combo box, the event will highlight the component in the Human Information Processing. The guidelines will be displayed at the guidelines screen. Selecting Combination command button will enable user to see the combo box for media type combinations.

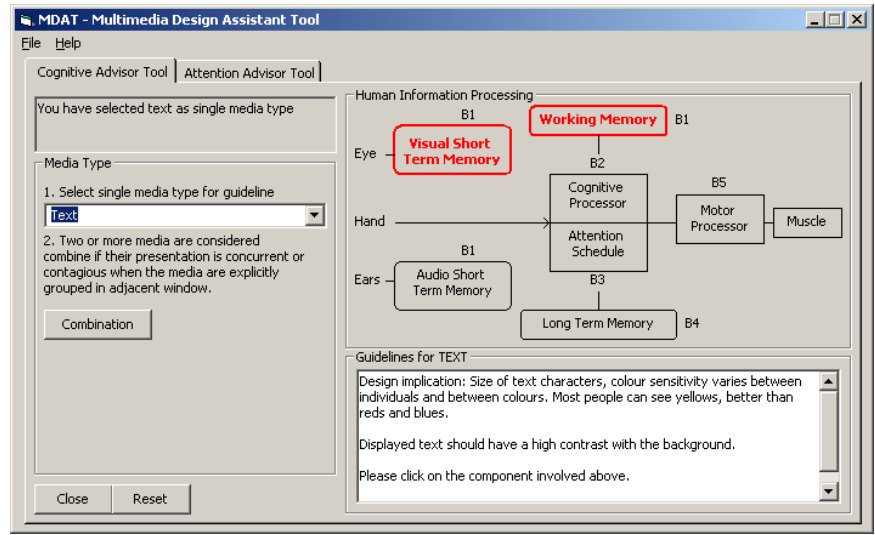

Figure 5: Shows the implemented function of Cognitive Advisor Tool.

The MDAT is characterised by the following features - a basic communication infrastructure, such as components/objects and the advisor that can interact with the designers; an algorithm, which matches operations based on syntactic criteria (media types); and semantic criteria (pre- and post conditions) is used in the tool. The MDAT's user interface is all about responding to events and user actions.

\section{Summary}

This research has introduced the concept of cognitive human information processing. The user requirements specification produced for a functional and 
usable design tool allows for the creation of attention directing applications, thereby constituting useful input for future development of similar or more advanced tool. In conclusion, the psychological background model has the power and flexibility to become an effective tool for designers. This research has made the first step to the approach of using psychological knowledge to match multisensory interaction used with human mental activity. However, future research and implementation is certainly required so that the opportunities are transformed into successful practices to be used by all.

\section{References}

[1] Chen, Qiyang and Norcio, A.F., (2001). "Knowledge Engineering in Adaptive Interface and User Modelling”, Human Computer Interaction: Issues and Challenges, pp 113-133.

[2] Jae Eun Shin (2002). "Evaluating the Effectiveness of a Multimedia Advisor Tool", Computer Department, UMIST, United Kingdom.

[3] Kieras, D.E., and Mayer, D.E (1997). "An Overview of the EPIC Architecture for Cognition and Performance with Application to Human Computer Interaction”. In John M. Caroll, Human-Computer Interaction in the New Millennium, Vol.12, No.4, pp 391-438.

[4] Klattzky, R.L (1980). Human Memory: Structure and Processes, Second Edition. San Francisco: HW. Freeman Co.

[5] Kolko, Jon (2004). "Mixing Disciplines in Anticipation of Convergence: A Curriculum for Teaching Interaction Design to Industrial Designers" in ACM's Interactions, V11/issue 4.

[6] Myers, B., Hudson, Scott E. \& Pausch R. (2000). "Past, Present, and Future of User Interface Software Tools". ACM Transactions on Computer-Human Interaction, Vol. 7, No. 1, March 2000. In John M. Caroll, Human-Computer Interaction in the New Millennium, pp 213-233.

[7] Shneiderman B. (1998). "Designing the User Interface: Strategies for Effective Human-Computer Interaction", $3^{\text {rd }}$ edition.

[8] Sutcliffe, A.G. (2000). "On the Effective and Reuse of HCI Knowledge”. ACM Transactions on Computer-Human Interaction, Vol. 7. No. 1, March 2000. In John M. Caroll, Human-Computer Interaction in the New Millennium, pp 3-29.

[9] Tom Vavik (2001). "Human Factors and Interaction Design for Industrial Designers" at http://www.intermedia.uio.no/seminarer/designingdesign/ Human factors.htm

[10] Vanderheiden Gregg C. (2001). "Development of generic accessibility/ability usability design guidelines for electronic and information technology products. Paper presented at 1st International Conference on Universal Access in Human-Computer Interaction (HCI), New Orleans, LA, August, 2001. 